\title{
Passive Torque Regulation in an Underactuated Flapping Wing Robotic Insect
}

\author{
P. S. Sreetharan and R. J. Wood \\ School of Engineering and Applied Science \\ Harvard University \\ Cambridge, Massachusetts 02138 \\ pratheev@post.harvard.edu
}

\begin{abstract}
Recent developments in millimeter-scale fabrication processes have led to rapid progress towards creating airborne flapping wing robots based on Dipteran (two-winged) insects. Previous work to regulate reaction forces and torques generated by two flapping wings has largely focused on wing trajectory control. An alternative approach introduces additional degrees of freedom to the wing flapping mechanism to passively regulate these forces and torques. The resulting 'mechanically intelligent' devices can execute wing trajectory corrections to realize desired body forces and torques without the intervention of an active controller.

This paper describes an insect-scale flapping wing aeromechanical structure consisting of a piezoelectric bimorph power actuator, an underactuated transmission mechanism, and passively rotating wings. The transmission is designed to passively modulate wing stroke velocity to eliminate the net roll torque imparted to the airframe.

The system is modeled as having four degrees of freedom driven open-loop by a single power actuator. The theoretical model predicts lift-generating wing trajectories as well as a passive reduction in roll torque experienced by the airframe. An at-scale structure constructed using Smart Composite Microstructure (SCM) fabrication techniques provides experimental support for the theoretical model.
\end{abstract}

\section{INTRODUCTION}

Advances in millimeter scale fabrication processes have enabled rapid progress towards the development of flapping wing micro air vehicles (FWMAVs) with system mass on the order of $100 \mathrm{mg}$ [1]. However, flight stability and control mechanisms for these mass and power limited systems remain active areas of research.

Investigation into the aerodynamics of biological insect flight has produced approximate aerodynamic models allowing computationally inexpensive prediction of aerodynamic forces and torques from wing trajectories [2], [3]. Accordingly, research into transmission and control mechanisms of flapping wing robotic insects has focused on control of wing trajectory. For example, the Berkeley Micromechanical Flying Insect (MFI) is a FWMAV platform with the ability to execute a range of predetermined wing trajectories using a fully actuated wing drive mechanism, neglecting elastic deformation of the transmission and wings [4]. In one notable exception, the Harvard Microrobotic Fly (HMF) has proven capable of realizing qualitatively biomimetic wing trajectories using passive compliance to allow variation of wing angles of attack [1]. The associated reduction in complexity has allowed this aeromechanical platform to achieve a lift to weight ratio greater than unity.

However, the benefits of underactuation and passive compliance can extend beyond simple reduction of mechanical complexity, in particular for devices in which the distribution of forces and torques is of fundamental importance. A ubiquitous example is the automobile differential, an underactuated mechanism commonly used to distribute engine power to two wheels. The differential incorporates an additional degree of freedom $q_{2}$ to balance the torque delivered to each wheel (see Fig. 1). The differential fundamentally operates on wheel torques instead of rotations; aided by passive mechanisms, the wheels can rotate along complex relative trajectories, maintaining traction on the ground without closed loop active control.

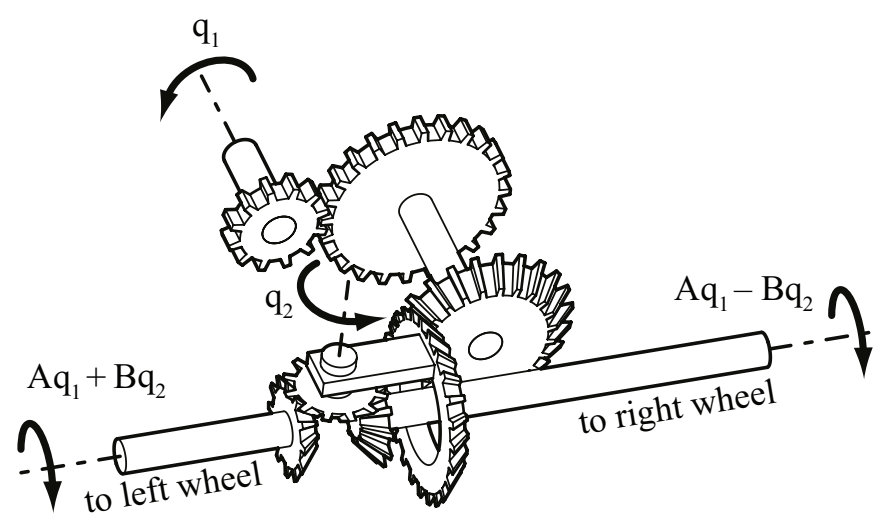

Fig. 1: A car differential balances output torques using an underactuated mechanism. Degree of freedom $q_{1}$ receives engine torque while $q_{2}$ is unactuated.

Previous work has introduced the concept of Passive Aeromechanical Regulation of Imbalanced Torques (PARITy) in the context of insect-scale FWMAV design [5]. Embodying the PARITy concept, the 'Drag PARITy' is an underactuated two degree of freedom FWMAV transmission that, analogous to an automobile differential, passively distributes power from a single actuator to balance torques delivered to two wings. Previous work has demonstrated its effectiveness within a planar two degree of freedom system with fixed $90^{\circ}$ wing angles of attacks. Though the transmission was shown to 
passively balance drag induced roll torques, a fixed $90^{\circ}$ angle of attack prevents the wings from generating lift.

This paper describes a lift-generating FWMAV design integrating passively rotating wings with the Drag PARITy transmission. Variation of wing angle of attack is achieved by incorporating a compliant 'wing hinge' (Fig. 3c) into the wing, similar to the approach taken by [1]. The complete design described in this paper has four degrees of freedom, a significant increase in dynamic complexity over the two degree of freedom system previously demonstrated in [5]. A single power actuator applies an oscillatory force, exciting motion in all four degrees of freedom through a variety of aerodynamic and inertial effects.

The underactuated flapping wing system is shown to execute stable, qualitatively biomimetic, lift-generating wing trajectories, indicating that the Drag PARITy is a viable transmission design for insect-scale FWMAVs. A theoretical model of the system is developed to investigate torque balancing characteristics in simulation. A control ('Uncut') trial with a nominally symmetric system demonstrates passive balancing of roll torques imparted from each wing, compensating for fabrication variation. In '1-Cut' and '2-Cut' trials, the system is simulated with successive removal of planform area from one wing (to provide an asymmetric disturbance) and is shown to continue successfully balancing roll torques, compensating for large inertial and aerodynamic wing asymmetries. By passively diverting more power to an underperforming wing, the design is also shown to indirectly compensate for imbalanced lift generation. Finally, an at-scale test device is constructed and observed to execute wing trajectories supporting theoretical predictions. Prior to describing the experiment, however, the PARITy methodology for FWMAV control which motivates this investigation will first be outlined.

\section{The PARITy Methodology}

Though it would allow for a highly capable FWMAV, fully-actuated high-bandwidth control of wing trajectories has not been achieved on a $100 \mathrm{mg}$ platform. Millimeter-scale fabrication techniques have not yet demonstrated the requisite complexity within mass constraints. Furthermore, power and mass constraints are likely to limit the bandwidth of electronic sensing and control systems on these lightweight platforms.

Acknowledging these limitations, research has been conducted into 'time-averaged' wing control, seeking to control average forces and torques by applying kinematic wing trajectory corrections on a long timescale (longer than a wing flapping period) [6]. Assuming that active control will not be attempted at short (sub-wingbeat) timescales, the question of the ideal short timescale behavior of a wing flapping mechanism is raised. Traditional kinematic control approaches tacitly assume that rigid specification of wing trajectory is a preferred short timescale behavior.

However, the specific wing trajectory executed is not fundamentally important to an FWMAV. Rather, an active flight control system for a robotic flier uses the wings as a tool to generate desired reaction forces and torques on its airframe.

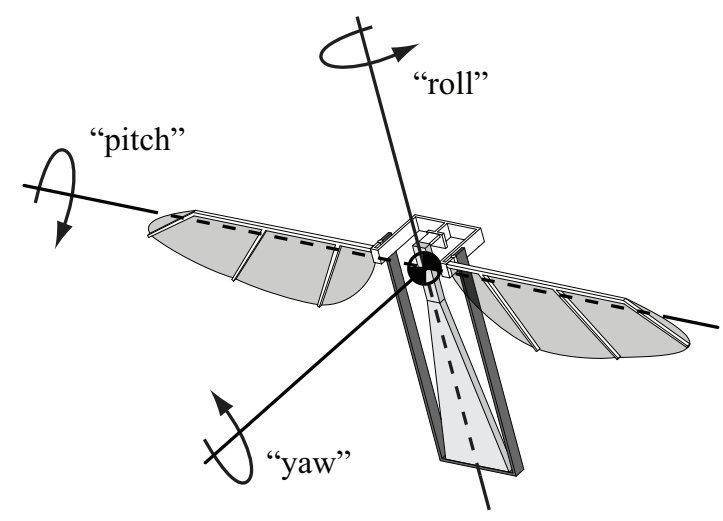

Fig. 2: Definition of roll, pitch, and yaw in the body frame.

Ideally, the wings should execute whatever trajectories are necessary to realize these desired forces and torques. ${ }^{1}$ A drivetrain that passively regulates these forces and torques at a short timescale may simplify a longer timescale flight controller.

This alternative short term behaviour is conjectured to produce systems that reject short timescale disturbances passively, alleviating requirements on active control systems. It is also expected to compensate for some fabrication asymmetries, passively realizing the necessary adjustments to wing trajectory. This feature is extremely attractive, since fabrication variation is a major concern for devices manufactured at the millimeter scale.

Under the PARITy methodology, long timescale control is achieved not by altering the wing trajectories directly, but by modulating the dynamics of the short timescale passive system. In the context of PARITy based FWMAV designs, control inputs would perturb the setpoint of short timescale system dynamics. For example, the 'Drag PARITy' drivetrain analyzed in this paper passively balances body roll torques imparted by each wing. However, actuation of an active control input could bias system dynamics such that the roll torque imparted by one wing is passively regulated to be $10 \%$ higher than that from the other wing. This local passive regulation may enable direct active force and torque control at long timescales, simplifying the control problem for mass-limited flapping wing aeromechanical platforms.

Such active control mechanisms are the subject of future work and will not be discussed in depth in this paper, but their brief mention serves to motivate the detailed analysis of simpler PARITy drivetrains without control capability. The following sections analyze a specific FWMAV system introducing passively rotating wings to an actuated Drag PARITy transmission.

\footnotetext{
${ }^{1}$ The specific trajectory may be important for efficiency concerns, but is irrelevant for the purposes of stabilizing and controlling the airframe.
} 


\section{THE MECHANISM}

\section{A. Actuation}

Piezoelectric actuation has been chosen due to its high bandwidth and high power density [7]. The actuator is a bimorph PZT cantilever, with a peak-to-peak actuation stroke of approximately $500 \mu \mathrm{m}$. The base of the cantilever is grounded to the FWMAV airframe, while the output is affixed to the transmission input (Fig. 3).

\section{B. The Drag PARITy transmission}

The Drag PARITy transmission is a millimeter scale planar linkage constructed using Smart Composite Microstructure (SCM) fabrication techniques [8]. Unidirectional carbon fiber beams form rigid links, while revolute joints are realized by polymer flexure interconnects. The transmission has a single actuated input $q_{1}$ and dual outputs driving the stroke angles of each wing. The right wing stroke angle $\phi^{R}$ is illustrated in Fig. 3c, while the left wing stroke angle $\phi^{L}$ (not shown) is the analogous angle on the opposing wing.
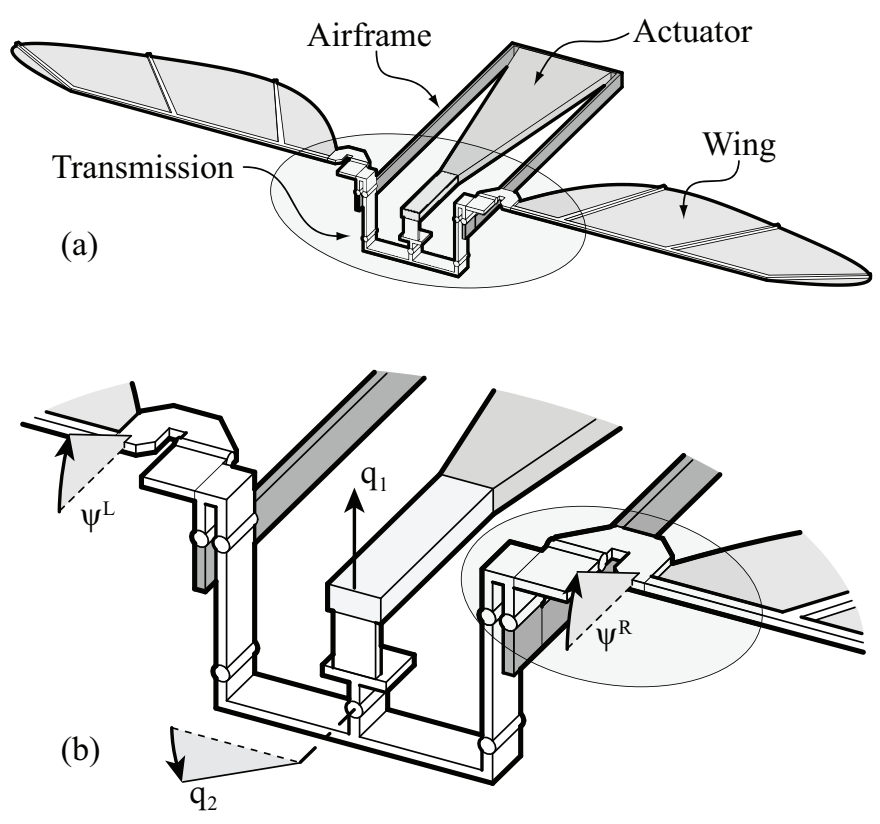

(b)

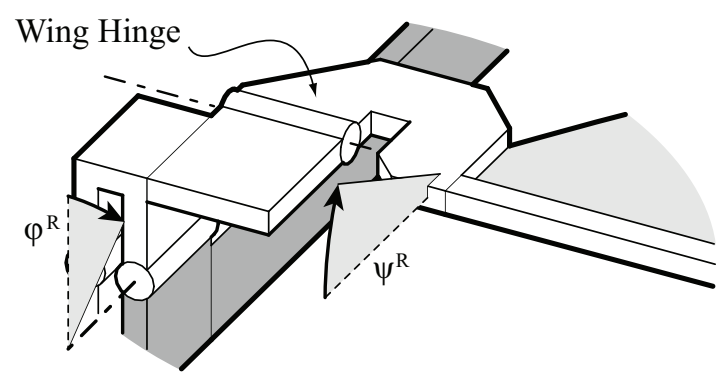

Fig. 3: (a) Diagram of the FWMAV design. (b) The four degrees of freedom $q_{1}, q_{2}, \psi^{L}$, and $\psi^{R}$ with respect to airframe ground. (c) A view of the shoulder clarifying rotation angle $\psi^{R}$. Right wing stroke angle $\phi^{R}$ can be determined from $q_{1}$ and $q_{2}$, as can $\phi^{L}$ of the left wing (not shown).
The transmission mechanism has two degrees of freedom; referring to Fig. 3b, $q_{1}$ is actuated and allows power to be injected into the system, while $q_{2}$ is passively determined. The degree of freedom $q_{2}$ couples the upstroke of one wing to the downstroke of the other, allowing the mechanism to passively modulate wing stroke velocities to balance the roll torques imparted by the wings on the airframe. An invertible kinematic mapping relates $q_{1}$ and $q_{2}$ to $\phi^{R}$ and $\phi^{L}$; either pair of coordinates can be used to describe the configuration of the transmission. A more detailed description of this mechanism is available in [5].

\section{Wings}

Wings consist of a $1.5 \mu \mathrm{m}$ polyester membrane supported by carbon fiber venation, shown in Fig. 4. Fabricated wings have masses under $1 \mathrm{mg}$ and are effectively rigid plates, exhibiting limited deformation while flapping. In an approach pioneered by [1], each wing is attached to a transmission output in series with a polymer flexure 'wing hinge' that allows the rigid wing to passively rotate around its longitudinal axis (see Fig. 3c). Compliance around the wing hinge axis allows the angle of attack of each wing to vary passively while flapping.

\section{Theoretical Simulation}

\section{A. Actuation}

Actuator drive voltage is the single input to the simulation model. Using results from a laminate plate theory analysis, the first bending mode of the cantilever power actuator has been modeled as a grounded spring in parallel with a voltageproportional force [7]. The cantilever beam has a linear spring constant of $467 \mathrm{mN} / \mathrm{mm}$, and under a $100 \mathrm{~V}$ amplitude sinusoidal drive signal, the actuator exerts a $120 \mathrm{mN}$ amplitude sinusoidal force. The drive signal is applied at $110 \mathrm{~Hz}$, near mechanical resonance to increase stroke amplitude and limit reactive power.

\section{B. Mechanical model}

The transmission mechanism along with the wing hinge has been treated using a pseudo rigid body model [9]. All carbon fiber links are assumed to be infinitely stiff, while polymer flexure interconnects have been modeled as perfect revolute joints in parallel with linear torsion springs. Spring constants for the transmission joints and wing hinges have been calculated using classical beam theory, and no damping or other internal loss mechanisms have been modeled.

The wings themselves are the only significant inertias within the system and are the only inertias considered in the model. The mass of the SCM linkage mechanism is neglected. Though the piezoelectric actuator mass is significant, due to the large transmission ratio the effective inertia of the actuator is negligible and has been omitted from the model.

The final theoretical system has four degrees of freedom: two are contained within the Drag PARITy transmission, while the two wings each add a degree of freedom from their respective wing hinges. The orientation of each wing can be fully described by the angle of the corresponding transmission 

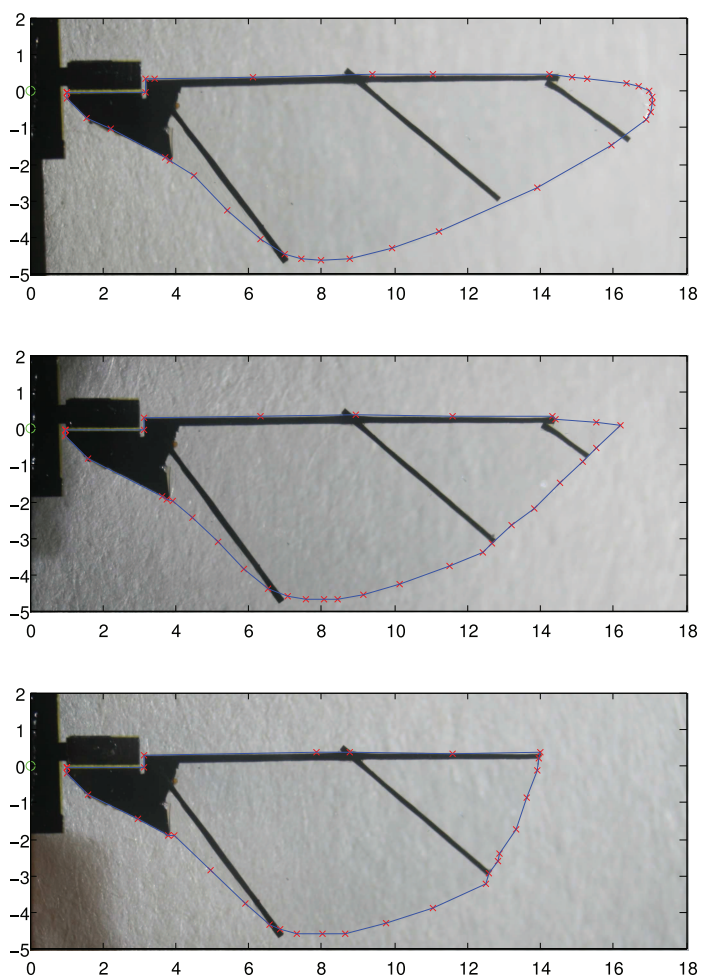

Fig. 4: The wing with membrane outline indicated for the Uncut, 1-Cut, and 2-Cut trials, from top to bottom. Axis units are in mm. For inertial components in Table I, The $z$ and $x$ coordinate axes correspond to horizontal and vertical image axes, respectively.

\begin{tabular}{c|cccc} 
Wing & Left & Right & Right & Right \\
Trial & All & Uncut & 1-Cut & 2-Cut \\
\hline$I_{x x}$ & 49.0 & 47.1 & 40.6 & 32.8 \\
$I_{y y}$ & 50.5 & 48.6 & 42.0 & 34.1 \\
$I_{z z}$ & 1.56 & 1.49 & 1.43 & 1.29 \\
$I_{x z}$ & 4.20 & 3.87 & 3.38 & 2.97 \\
$\Omega_{1}$ & 46.8 & 38.2 & 28.7 & 21.2 \\
$\Omega_{2}$ & 0.587 & 0.438 & 0.419 & 0.387 \\
$\Omega_{3}$ & 17.5 & 13.5 & 10.6 & 9.10 \\
$\Omega_{4}$ & 0.712 & 0.952 & 0.787 & 0.691
\end{tabular}

TABLE I: Inertial and aerodynamic parameters used for the left and right wings for the Uncut, 1-Cut, and 2-Cut trials. All values have units of $\mathrm{mg} \cdot \mathrm{mm}^{2}$. The coordinate frame for inertial components is described in Figure 4.

output (the 'stroke angle' $\phi$ ) and the deflection angle of the wing hinge (the 'rotation angle' $\psi$ ), illustrated for the right wing in Fig. 3c.

\section{Aerodynamic model}

Aerodynamic effects have been simulated using a model derived from the blade element method, assuming a perfectly rigid wing planform. As modeled, lift and drag torques are proportional to $\dot{\phi}^{2}$, the square of stroke velocity. Averaged lift and drag coefficients, strong functions of the rotation angle $\psi$, were taken from experimental data collected from dynamically scaled models of a fruit fly (Drosophila melanogaster) wing flapping in mineral oil [2]. Calculation of wing rotational moments, important for realizing passive wing rotation, relies on additional experimental work quantifying a non-dimensional center of pressure location $\hat{d}_{c p}$ of fruit fly wings [3], [10]. Rotational damping, proportional to $\dot{\psi}^{2}$, the square of wing rotational velocity, has been modeled in accordance with experimental and theoretical work on tumbling plates [11].

The complete aerodynamic model can be distilled into the following four aerodynamic moments applied to each wing:

$$
\begin{aligned}
M_{N} & =-\Omega_{1} \operatorname{sgn}(\dot{\phi}) \dot{\phi}^{2} C_{N}(\psi) \\
M_{T} & =-\Omega_{1} \operatorname{sgn}(\dot{\phi}) \dot{\phi}^{2} C_{T}(\psi) \\
M_{r d} & =-\Omega_{2} \operatorname{sgn}(\dot{\psi}) \dot{\psi}^{2} C_{r d} \\
M_{r} & =-\left(\Omega_{3} \hat{d}_{c p}(\psi)-\Omega_{4}\right) \operatorname{sgn}(\dot{\phi}) \dot{\phi}^{2} C_{N}(\psi)
\end{aligned}
$$

In the previous set of equations, $M_{T}$ acts about an axis perpendicular to the wing plane and is the result of aerodynamic forces acting in the wing plane. $M_{r}$ and $M_{r d}$ are the rotational and rotational damping moments, respectively, both acting on the wing around the wing hinge axis. $M_{N}$ acts about an axis perpendicular to both the wing plane normal and the hinge axis, and results from aerodynamic forces normal to the wing. The three aerodynamic coefficients, related to tangential $\left(C_{T}\right)$, normal $\left(C_{N}\right)$, and rotational damping $\left(C_{r d}\right)$ aerodynamic forces, are described in [10].

The parameters $\Omega_{1}, \Omega_{2}, \Omega_{3}$, and $\Omega_{4}$ have units of $\mathrm{mg} \cdot \mathrm{mm}^{2}$ and can be calculated from the air density $\rho$ and the specific wing morphology (see Table I for calculated values). Related work has produced extensive experimental data verifying that this aerodynamic model adequately describes passive rotation of a single wing executing a predetermined stroke angle trajectory, along with generated lift forces [10]. This referenced work contains a detailed description of the aerodynamic model briefly summarized here.

\section{Mathematical formulation}

For the theoretical model, the four coordinates specifying the device configuration were taken to be the left and right wing stroke angles $\left(\phi^{L}\right.$ and $\phi^{R}$, respectively) along with the left and right wing rotation angles $\left(\psi^{L}\right.$ and $\psi^{R}$, respectively). These four quantities and their time derivatives $\dot{\phi}^{L}, \dot{\phi}^{R}, \dot{\psi}^{L}$, and $\dot{\psi}^{R}$ form the full eight element state vector of the dynamic system.

The body inertia of a robotic $100 \mathrm{mg}$ FWMAV is assumed to be orders of magnitude larger than the wing inertias. This assumption is representative of many biological insects, though some exceptions exist (e.g. butterflies). Accordingly, the body frame has been treated as an inertial reference frame for the purpose of predicting wing dynamics. This assumption accurately represents the grounded-airframe experiment undertaken in Section VI. Furthermore, theoretical wing dynamic predictions are not expected to be impacted significantly by the non-inertial nature of the body frame of a free flying FWMAV.

The equations of motion for the wings were derived from an Euler-Lagrange formulation assuming a fixed body frame. 


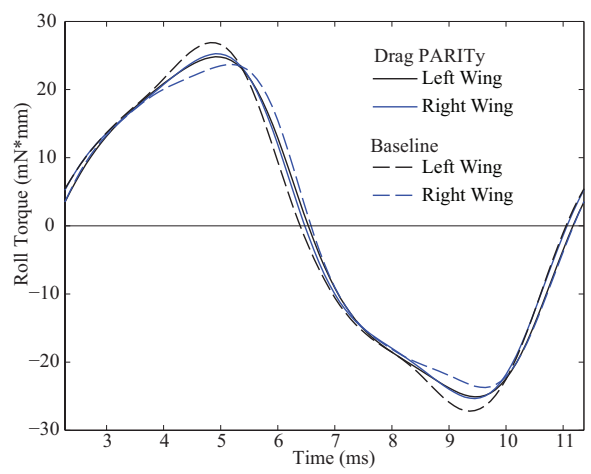

(a)

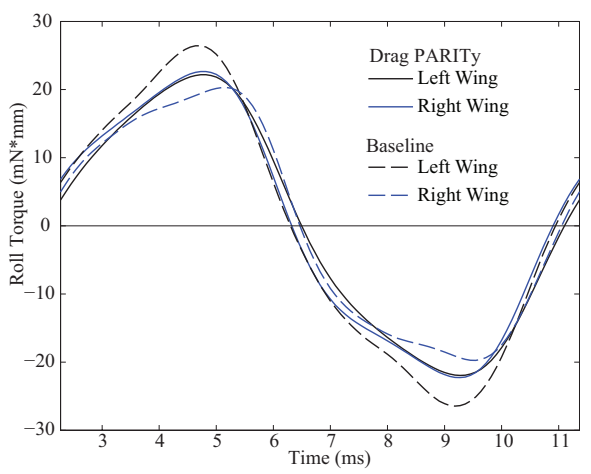

(b)

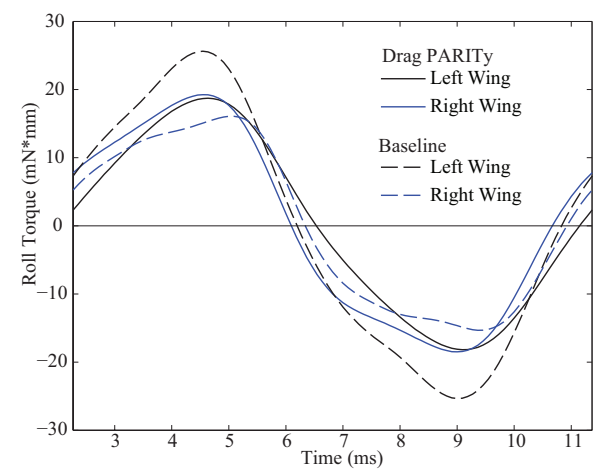

(c)

Fig. 5: Theoretical roll torque experienced by the airframe in the (a) Uncut, (b) 1-Cut, and (c) 2-Cut trials.

Since the only modeled inertias in the system are those of the two wings, the form of kinetic energy $T$ is straightforward:

$$
T=\frac{1}{2}\left(\vec{\omega}^{L}\right)^{T} \boldsymbol{I}^{L} \vec{\omega}^{L}+\frac{1}{2}\left(\vec{\omega}^{R}\right)^{T} \boldsymbol{I}^{R} \vec{\omega}^{R}
$$

In the preceding equation, $\boldsymbol{I}^{L}$ and $\boldsymbol{I}^{R}$ are the inertial tensors of each wing, constant in the wing frame and calculated about an origin defined by the closest point on the wing hinge axis to the shoulder axis (see Table I for calculated values). The small shoulder offset of the Drag PARITy transmission has been neglected, thus no translational kinetic energy terms are present. The term $\vec{\omega}^{L}$ is the angular velocity of the left wing, a function of $\phi^{L}, \psi^{L}, \dot{\phi}^{L}$, and $\dot{\psi}^{L}$. An analogous statement applies to the right wing angular velocity $\vec{\omega}^{R}$.

The potential energy $V$ has the following form:

$$
V=\frac{1}{2} \sum_{i=1}^{9} k_{i} \gamma_{i}^{2}+\frac{1}{2} k_{a} q_{1}^{2}
$$

The full device contains nine polymer flexure joints: two wing hinges along with seven internal to the Drag PARITy transmission. The quantities $\gamma_{i}$ represent the angular deflection of each flexure joint, functions of $\phi^{L}, \psi^{L}, \phi^{R}$, and $\psi^{R}$. The constants $k_{i}$ represent the linearized torsional spring constants for each polymer flexure joint. The constant $k_{a}$ is a linear spring constant describing the restoring force of the actuator in response to its linear deflection $q_{1}$, itself a function of $\phi^{L}$ and $\phi^{R}$.

The Lagrangian $L$ is defined as $L=T-V$, and the equations of motion are derived from the Euler-Lagrange equations for each of the four coordinates $p_{i} \in\left\{\phi^{L}, \psi^{L}, \phi^{R}, \psi^{R}\right\}$ :

$$
\frac{\mathrm{d}}{\mathrm{d} t} \frac{\partial L}{\partial \dot{p}_{i}}-\frac{\partial L}{\partial p_{i}}=\tau_{i}
$$

Actuation force as well as aerodynamic torques appear in the model as generalized forces $\tau_{i}$. Actuation occurs along $q_{1}$ (see Fig. 3) and aerodynamic torques are more naturally calculated in the wing frame, so the appropriate Jacobians have been used to map these forces onto the configuration variables.

All necessary Jacobian matrices and partial derivatives have been derived in closed analytical form for use within the model, but the details have been omitted for brevity. The four $2^{\text {nd }}$ order differential equations produced from (7) were expressed as a first order system of eight coupled nonlinear differential equations. All theoretical results for the Drag PARITy design are the result of numerically integrating these differential equations using a Runge-Kutta based method as implemented by the MATLAB function ode 45 .

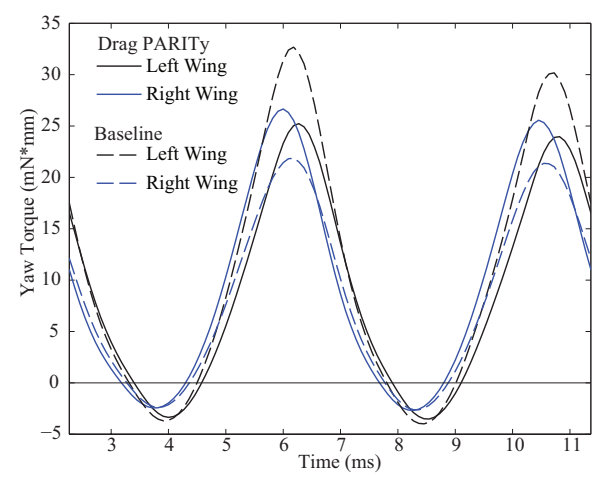

Fig. 6: Yaw torque in the 1-Cut Trial.

The theoretical dynamics model has been used to investigate the reaction torque regulating properties of the system in response to wing asymmetry. The system was compared with a baseline design in which the Drag PARITy transmission has been replaced with a conventional transmission characterized by fully actuated wing stroke angles. This baseline design is realized by freezing the degree of freedom $q_{2}$ of the Drag PARITy to $q_{2}=0$ (equivalent to the constraint $\phi^{L}=\phi^{R}$ ). This constraint is accommodated by introducing a time-dependent Lagrange multiplier to the Lagrangian:

$$
L=T-V+\lambda\left(\phi^{L}-\phi^{R}\right)
$$

In the modified equations of motion given by (7), $\lambda(t)$ represents an internal constraint force and is calculated algebraically at each timestep to satisfy the kinematic constraint. It is to be noted that this baseline three degree of freedom design is identical to that of the HMF [1]. 
A control trial, which will be called the 'Uncut' trial, was simulated using a structure mechanically and aerodynamically symmetric to the tolerances achievable with the SCM manufacturing process. Two additional trials were undertaken with intentionally asymmetric wing parameters, realized by removing successive amounts of planform area from the distal extent of the right wing. These trials will be called the 1Cut and 2-Cut trials, respectively. Images of the right wing planform for all three trials are shown in Fig. 4. The left wing is nominally identical to the Uncut right wing for all three trials.

All wing parameters were measured directly from wings fabricated for the experimental test structure. Inertia tensors for the wing were constructed using a baseline mass measurement coupled with a photogrammetric process to determine the spatial distribution of wing mass. Aerodynamic parameters for the wings were calculated using the photogrammetrically determined wing planform areas shown in Fig. 4. Since both inertial and aerodynamic properties are highly sensitive to wing mounting accuracy, the photogrammetric techniques were conducted in situ to avoid disturbing the device. Fabrication variation has resulted in measurable asymmetry even in the Uncut case, apparent in theoretical and experimental results. See Table I for all calculated inertial and aerodynamic parameters.

In all trials, the Drag PARITy design is observed to execute stable wing trajectories qualitatively similar to those executed by biological insects. Wing stroke angles $\phi^{L}$ and $\phi^{R}$ oscillate over approximately $100^{\circ}$ with a rotation angles $\psi^{L}$ and $\psi^{R}$ oscillating between $\pm 60^{\circ}$, approximately $90^{\circ}$ out of phase. These rotation angles correspond to an angle of attack $\alpha=90^{\circ}$ at stroke extents and $\alpha=30^{\circ}$ midstroke. Theoretical wing trajectories are plotted in Figs. 9a and 9c.

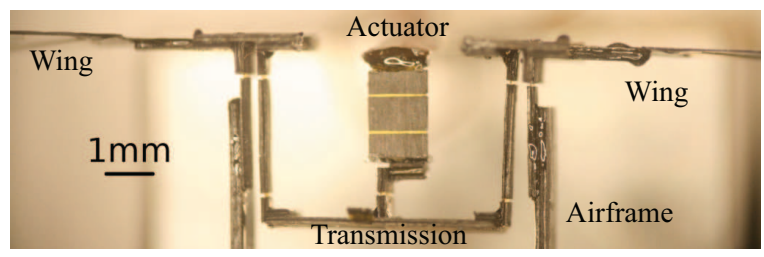

Fig. 7: Front view of the experimental device.

\section{PASSIVE BODY tORQUe REgUlation}

The Drag PARITy design distinguishes itself from the baseline design in the theoretical reaction torques imparted by the flapping wings on the airframe. The Drag PARITy is designed to balance the roll reaction torques imparted by the flapping wings. Fig. 5 plots the theoretical roll torques experienced by the body of a FWMAV using a Drag PARITy transmission compared to that experienced by a FWMAV using a conventional transmission. In all three trials, it is apparent that the Drag PARITy transmission has succeeded in balancing the roll torques experienced by the body due to each wing. The results are especially striking in the Uncut trial
(Fig. 5a), where the transmission has passively compensated for fabrication and assembly error present in the nominally symmetric structure.

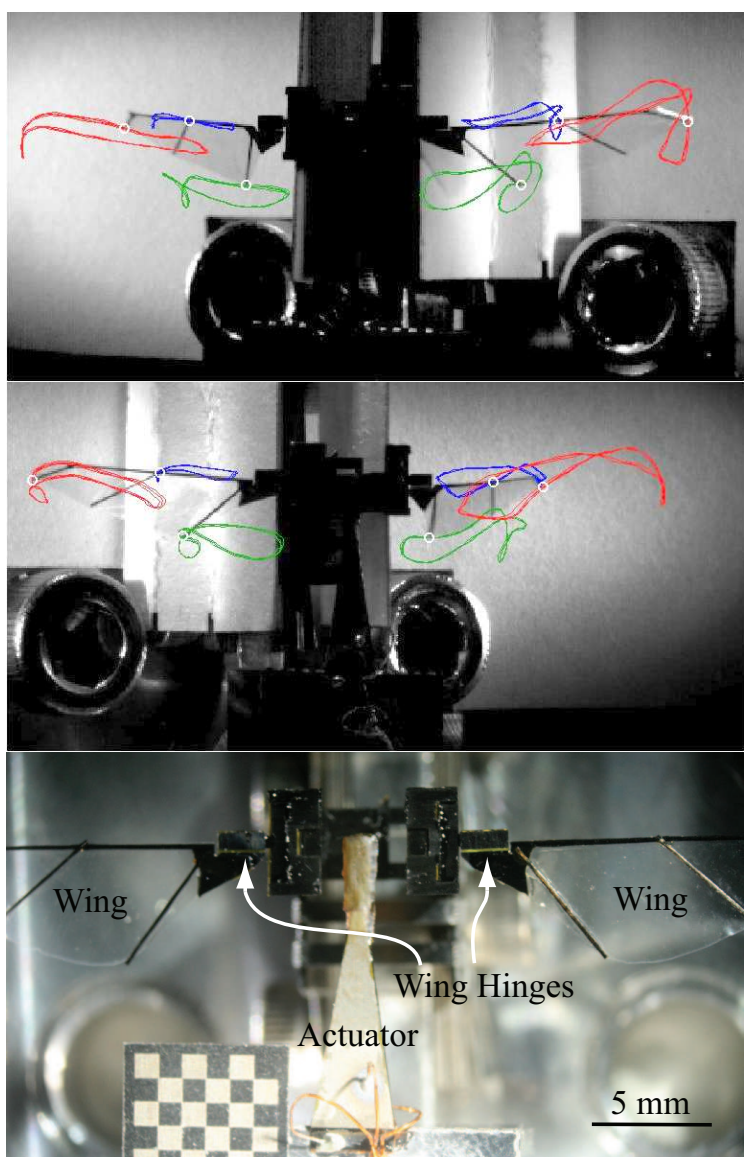

Fig. 8: Upper images are synchronized frames from the two cameras during the Uncut trial. Tracked points are indicated along with their trajectories over the course of the video. Lower image illustrates test structure.

Another interesting result of this theoretical study is the indirect balancing of lift dominated yaw torques. The results for the 1-Cut trial are presented in Fig. 6. In the drastically asymmetric 1-Cut and 2-Cut trials, use of the Drag PARITy design reduces the large average yaw torque imparted on the airframe by $71 \%$ and $72 \%$ respectively. However, in the nominally symmetric Uncut case, the Drag PARITy did not reduce the small average yaw error torque.

\section{EXPERIMENTAL VERIFICATION}

After acquiring data for the Uncut trial, the right wing was cut in situ to conduct the 1-Cut and 2-Cut trials without perturbing the alignment of the wing on the transmission output. As previously mentioned, all dynamic wing parameters were measured without disturbing the device and are presented in Table I.

Two high speed video cameras were positioned such that each obtained a clear view of both wings over the entire flapping motion. Prior to acquiring video, the cameras were 


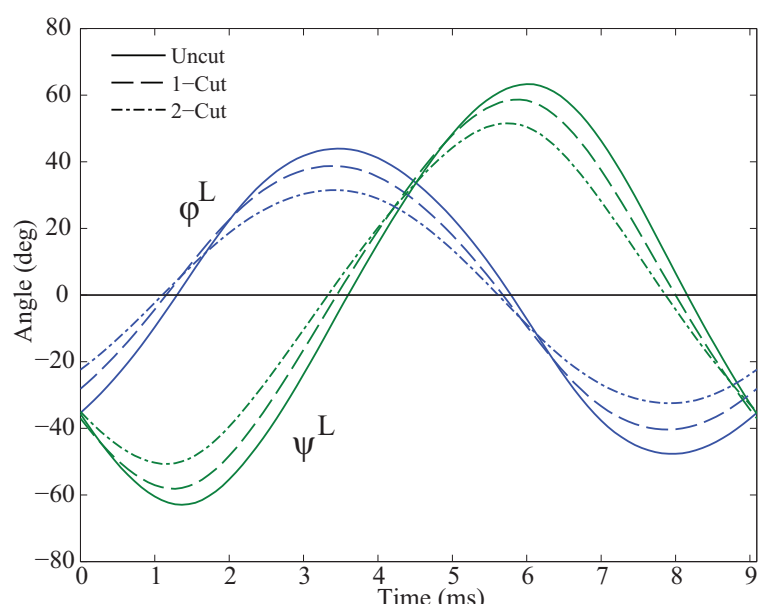

(a)

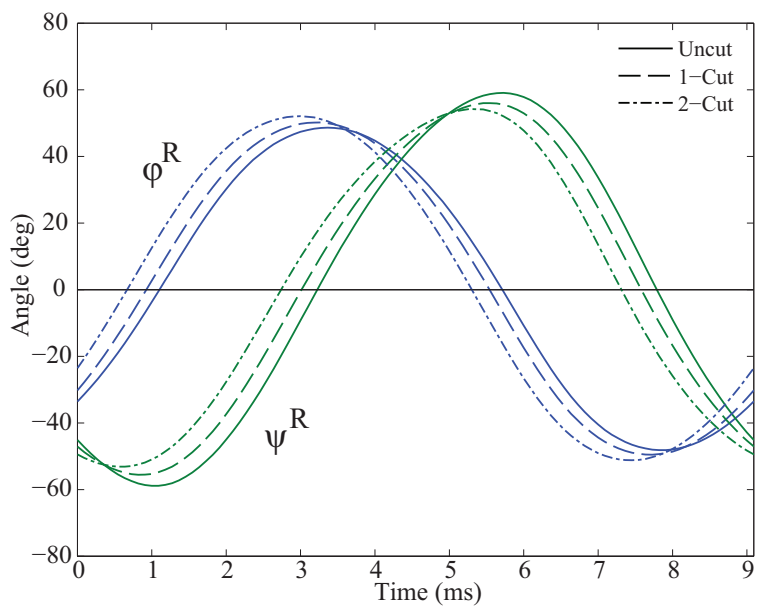

(c)

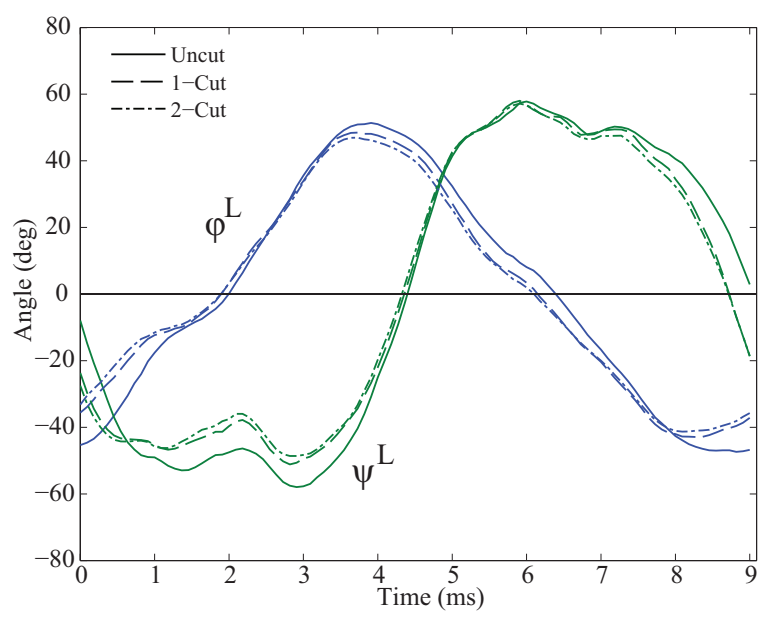

(b)

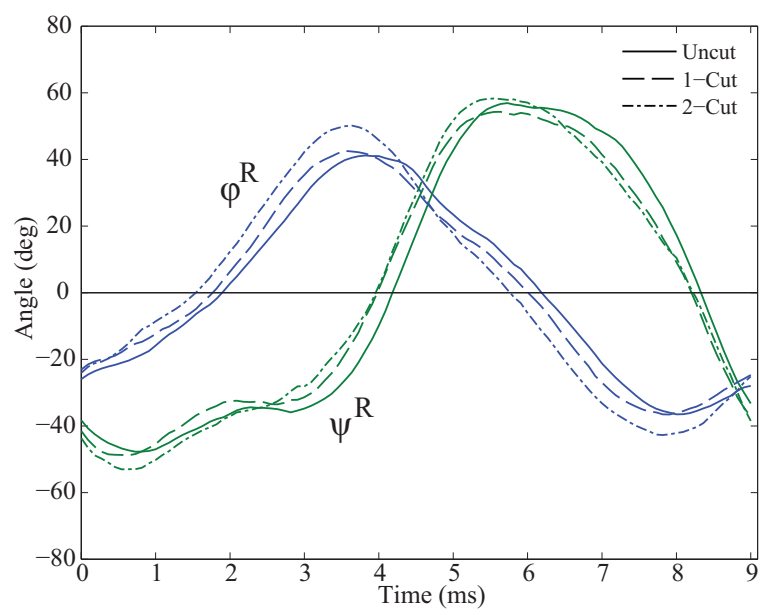

(d)

Fig. 9: Left wing trajectories (a) predicted by theory and (b) observed experimentally, along with (c) theoretical and (d) experimental right wing trajectories. Each plot shows results from the Uncut, 1-Cut, and 2-Cut trials.

calibrated using routines from the CalTech Camera Calibration Toolbox for MATLAB [12]. Once calibrated, the toolbox allows reconstruction of three dimensional coordinates of points identified in both camera views.

A $110 \mathrm{~Hz} 200 \mathrm{~V}$ (peak to peak) sinusoidal voltage was applied to the power actuator and synchronized high speed video was acquired from both video cameras at 10,000fps, or 91 frames per wingstroke period. Sample still frames are shown in Fig. 8.

Three easily distinguished features of the wing venation pattern were manually tracked across 300 frames for each trial. Identification of all three points in two camera views allows stereophotogrammetric reconstruction of the full wing orientation. The sinusoidal drive voltage applied to the actuator has been recorded and digitized at $5 \mathrm{kHz}$, synchronized with the high speed video stream.

The observed stroke and rotation angles are plotted as a function of time in Fig. 9, along with predictions produced by the theoretical model. Time synchronization has been achieved by aligning the theoretical and experimental drive signals, omitted from the plots for clarity. The functional form of the applied voltage signal as a function of time (in seconds) is:

$$
V(t)=100 \mathrm{~V}+100 \mathrm{~V} \cdot \sin (110 \cdot 2 \pi t)
$$

From Fig. 9, it is immediately apparent that the theoretical model accurately captures qualitative characteristics of the experimental model, with rotation angle $\psi$ exhibiting an approximately $90^{\circ}$ phase lag behind the stroke angle $\phi$. Furthermore, the theory also accurately predicts oscillation amplitudes from applied drive voltage, an achievement considering the complexity of this nonlinear dynamic system.

Theoretically predicted trends in wing trajectories as the right wing planform is altered are apparent in experimental data. The model predicts a monotonic increase in $\phi^{R}(t)$ amplitude as planform area is successively removed from the right wing, coupled with an associated decrease in the amplitude of $\phi^{L}(t)$. This trend is reflected in the experimental data as the Drag PARITy transmission passively diverts additional power to the underperforming right wing. The model also predicts a 
successive decrease in the amplitudes of both wing rotations $\psi^{L}(t)$ and $\psi^{R}(t)$ as wing membrane is removed. This trend is apparent in the observed trajectory of $\psi^{L}(t)$, though somewhat ambiguous in the observed trajectory of $\psi^{R}(t)$.

Among features not predicted by this simulation model are the square-wave appearance of observed wing rotations and the complex non-sinusoidal details of stroke angle trajectories. In future work, it is hoped that these discrepancies will be reduced by a more detailed theoretical model including, for example, mechanical loss mechanisms and nonlinear descriptions of polymer flexures to better predict dynamic characteristics at large joint angles. The transmission design itself will be refined to limit unintended and difficult to model behavior. For example, one source of error in this experimental trial was off-axis transmission compliance, resulting in measurable deviation of the wings from their mean stroke planes.

\section{ViI. CONCLUSion And Future Work}

This paper has presented further evidence supporting the utility of passive underactuated mechanisms in FWMAVs. Significantly extending previous work, the load balancing Drag PARITy transmission has been shown to be compatible with longitudinally compliant wing hinges allowing passive variation of wing angle of attack. The resulting singly actuated four degree of freedom system has been shown to execute stable qualitatively biomimetic flapping wing trajectories well described by the associated theoretical model. Furthermore, the Drag PARITy transmission is shown to maintain its load balancing capabilities, passively altering wing trajectories so as to balance roll torques experienced by the FWMAV airframe.

Future work exploring the PARITy methodology will proceed along two parallel tracks:

1) Demonstrating long timescale control mechanisms

2) Expanding passive regulation to larger subsets of body forces and torques

As previously mentioned, long timescale control in PARITy enabled FWMAVs will be achieved not by direct modulation of wing kinematic trajectories, but by active modification of system dynamics. For example, the Drag PARITy transmission described in this paper exhibits short timescale dynamics that balance roll torques from each wing. An active control input could be introduced to bias these dynamics such that they passively regulate the ratio of roll torques $\tau_{\text {roll }}^{L}$ and $\tau_{\text {roll }}^{R}$ from the left and right wings, respectively, to a specified setpoint $q_{3}:$

$$
\tau_{\text {roll }}^{L} / \tau_{\text {roll }}^{R}=q_{3}
$$

Note that $q_{3}$ is fixed at unity for the simple Drag PARITy transmission. A variety of dynamic parameters within the transmission, such as spring constants and link lengths, can be actively modulated at long timescales to realize this biased short timescale behavior. The potential for simple control relationships such as (10), bypassing wing kinematics to directly concern airframe forces and torques, is an exciting result of the PARITy methodology. Demonstration of such control features will motivate one track of future work.

A second research track involves introducing alternative or additional passive degrees of freedom to an FWMAV drivetrain to regulate different or expanded subsets of the body forces and torques produced by the wings. The Drag PARITy drivetrain is a mechanically intelligent device that has demonstrated regulation of body roll torques, arising in part from aerodynamic drag. In one nascent concept, careful introduction of passive features may enable an FWMAV drivetrain that directly regulates yaw torques arising from aerodynamic lift in addition to roll torques. The design space of such mechanically intelligent structures is vast, and future work will attempt to produce a variety of force and torque regulating FWMAV structures.

The PARITy methodology has the potential to simplify flight control of insect-scale robotic FWMAVs. It is hoped that future research into this novel methodology will provide tools to increase aerodynamic performance and reduce requisite system complexity, hastening the arrival of an autonomous 100mg-scale robotic FWMAV.

\section{ACKNOWLEDGEMENT}

The authors gratefully acknowledge support from the National Science Foundation (Award No. CMMI-07466 38). Any opinions, findings and conclusions or recommendations expressed in this material are those of the authors and do not necessarily reflect those of the National Science Foundation.

\section{REFERENCES}

[1] R. J. Wood, "The first takeoff of a biologically inspired at-scale robotic insect," IEEE Trans. Rob., vol. 24, pp. 341-347, 2008.

[2] M. H. Dickinson, F. O. Lehmann, and S. P. Sane, "Wing rotation and the aerodynamic basis of insect flight," Science, vol. 284, pp. 1954-1960, 1999.

[3] W. B. Dickson, A. D. Straw, C. Poelma, and M. H. Dickinson, "An integrative model of insect flight control," in Proc. AIAA Aerospace Sciences Meeting and Exhibit, Reno, NV, January 2006.

[4] R. S. Fearing, K. H. Chiang, M. H. Dickinson, D. L. Pick, M. Sitti, and J. Yan, "Wing transmission for a micromechanical flying insect," J. Micromechatronics, vol. 1, no. 3, pp. 221-237, 2001.

[5] P. S. Sreetharan and R. J. Wood, "Passive aerodynamic drag balancing in a flapping wing robotic insect," J. Mech. Des., vol. 132, 2010.

[6] X. Deng, L. Schenato, and S. Sastry, "Flapping flight for biomimetic robotic insects: Part ii-flight control design," IEEE Trans. Rob., vol. 22, no. 4, pp. 789-803, 2006.

[7] R. J. Wood, E. Steltz, and R. S. Fearing, "Optimal energy density piezoelectric bending actuators," Sensors \& Actuators: A. Physical, vol. 119, no. 2, pp. 476-488, 2005.

[8] R. J. Wood, S. Avadhanula, R. Sahai, E. Steltz, and R. S. Fearing, "Microrobot design using fiber reinforced composites," J. Mech. Des., vol. 130, 2008.

[9] L. L. Howell, Compliant Mechanisms. John Wiley and Sons, Inc., 2001.

[10] J. P. Whitney and R. J. Wood, "Aeromechanics of passive rotation in flapping flight," J. Fluid Mech., vol. In Press, 2010.

[11] A. Andersen, U. Pesavento, and Z. Wang, "Unsteady aerodynamics of fluttering and tumbling plates," J. Fluid Mech., vol. 541, pp. 65-90, 2005.

[12] J. Y. Bouguet, "Camera calibration toolbox for matlab," 2008, http://www.vision.caltech.edu/bouguetj/calib_doc/index.html. 